\title{
98. A Note on Non-Desargues Projective Plane
}

\author{
By Makoto Iто
}

(Comm. by K. KUNUGI, M.J.A., July 12, 1958)

Recently the subject of non-Desargues projective plane which satisfies the harmonic point axiom was prosperously studied. For instance, V. Havel [1] and N. S. Mendelsohn [2] discussed about this problem.

In this note, we shall prove the following fundamental theorem by using the technique of N. S. Mendelsohn [2].

The little Desargues' theorem is valid in any projective plane which satisfies the axiom of the fourth harmonic point. Its converse is also true.

The proof of the property has not been given by him.

Proof. In any projective plane which satisfies the axiom of the fourth harmonic point, there exists the mapping called elation (see N. S. Mendelsohn [2, p. 543]).

Let $l$ be any line and $O$ be any point in $l$ (Fig. 1). Let $A$ and $A^{\prime}$ be any two points collinear with $O$. Hence, there exists Elat $(O, l$; $A \rightarrow A^{\prime}$ ).

Let $B, C$ be any two points distinct from each other and not on $A A^{\prime}$ and not on $l$. By Elat $\left(O, l ; A \rightarrow A^{\prime}\right)$ points $C$ and $B$ will be mapped onto points $C^{\prime}$ and $B^{\prime}$ respectively. Then $C A$ meets $C^{\prime} A^{\prime}$ at $B^{\prime \prime}$ on $l$ and $A B$ meets $A^{\prime} B^{\prime}$ at $C^{\prime \prime}$ on $l$. $C^{\prime} B^{\prime}$ is the image of $C B$ which will be obtained by Elat $\left(O, l ; C \rightarrow C^{\prime}\right)$ or Elat $\left(O, l ; B \rightarrow B^{\prime}\right)$. Hence $C B$ meets $C^{\prime} B^{\prime}$ at $B^{\prime \prime}$ on $l$.

Conversely, in any projective plane the "little Desargues theorem" is equivalent to its converse, so it is sufficient to show by its one side.

According to the harmonic

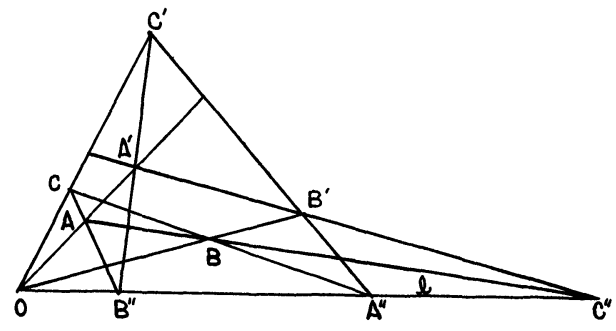

Fig. 1

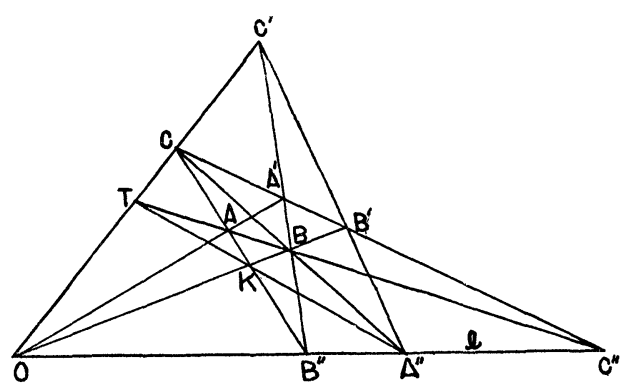

Fig. 2 diagram in his paper, let $A^{\prime \prime}, C^{\prime \prime}, O$ be any three points in line $l$ (Fig. 2). Let $C^{\prime}$ be any point not on $l$. Let $C^{\prime \prime} B^{\prime} C$ be any line through $C^{\prime \prime}$ distinct from $l$ and not passing through $C^{\prime}$, the point $C$ being on $C^{\prime} O$ 
and $B^{\prime}$ on $C^{\prime} A^{\prime \prime}$. Let $O B^{\prime}$ intersect $C A^{\prime \prime}$ at $B$ and let $C^{\prime} B$ intersect $l$ at $B^{\prime \prime}$. Also, let $A^{\prime}$ be point of intersection of $C^{\prime \prime} C$ and $C^{\prime} B$. Then points $A^{\prime \prime}, B^{\prime \prime}, C^{\prime \prime}, O$ related by such a dirgram will be said to satisfy the relation $H\left(O, A^{\prime \prime} ; C^{\prime \prime}, B^{\prime \prime}\right)$.

By Elat $\left(C^{\prime \prime}, l ; B^{\prime} \rightarrow A^{\prime}\right)$ point $B$ will be mapped to its image. Let the image of $B$ be $A$. Then $A$ and $A^{\prime}$ are collinear with $O$. By little Desargues theorem $C A$ meets $C^{\prime} A^{\prime}$ at $B^{\prime \prime}$ on $l$. Let $T$ be point of intersection of $A C^{\prime \prime}$ and $C^{\prime} O$, and let $K$ be point of intersection of $T A^{\prime \prime}$ and $O B$. By Elat $(O, l ; T \rightarrow C) K$ will be mapped on $B . C^{\prime} A^{\prime}$ is the image of $C A$ which will be obtained by Elat $\left(O, l ; C \rightarrow C^{\prime}\right)$ or Elat $\left(O, l ; A \rightarrow A^{\prime}\right)$. $\quad C^{\prime}, A^{\prime}, B$ are collinear, so $C, A, K$ are collinear. Hence $C A$ is passing through $K$.

\section{References}

[1] V. Havel: Über die lokalen Spezialisierungen des Satzes vom vollständigen Viereck und des kleinen Desarguesschen Satzes, Czeko. Math. Jour., 7, 295-307 (1957).

[2] N. S. Mendelsohn: Non-Desarguesian projective plane geometries which satisfy the harmonic point axiom, Canadian Jour. Math., 8, no. 4 (1956). 